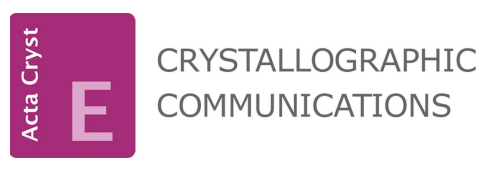

ISSN 2056-9890

Received 17 September 2015

Accepted 21 September 2015

Edited by D.-J. Xu, Zhejiang University (Yuquan Campus), China

Keywords: crystal structure; arylidene Meldrum's acid; 5-arylmethylene-2,2-dimethyl1,3-dioxan-4,6-dione; organic synthesis; intramolecular hydrogen bonding

CCDC reference: 1426237

Supporting information: this article has supporting information at journals.iucr.org/e

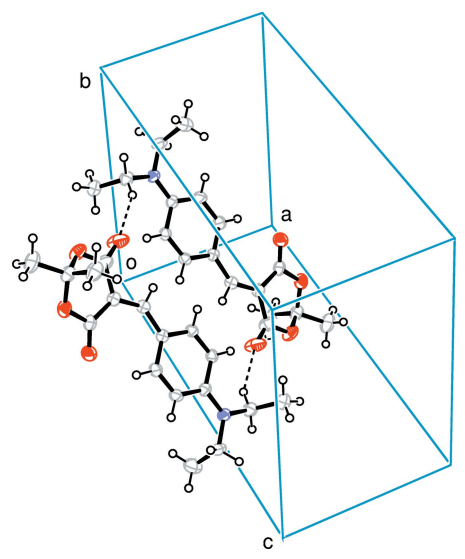

OPEN $\odot$ ACCESS

\section{Crystal structure of 5-[4-(diethylamino)benzyl- idene]-2,2-dimethyl-1,3-dioxane-4,6-dione}

\author{
Egija Stepina, ${ }^{a}$ Dmitrijs Stepanovs, ${ }^{a, b *}$ Inese Mierina ${ }^{\mathrm{a}}$ and Mara Jure ${ }^{\mathrm{a} *}$
}

${ }^{\text {a}}$ Faculty of Materials Science and Applied Chemistry, Riga Technical University, Str. P. Valdena 3/7, Riga, LV 1048, Latvia, and ${ }^{\mathbf{b}}$ Latvian Institute of Organic Synthesis, Str. Aizkraukles 21, Riga, LV 1006, Latvia. *Correspondence e-mail: d_stepanovs@osi.Iv, mara@ktf.rtu.Iv

The title compound, $\mathrm{C}_{17} \mathrm{H}_{21} \mathrm{NO}_{4}$, consists of substituted Meldrum's acid with a [4-(diethylamino)phenyl]methylidene fragment attached to the fifth position. The heterocycle assumes a distorted boat conformation. The planar part of heterocycle is almost coplanar with the benzene ring due to the presence of a long conjugated system in the molecule. This leads to the formation of $\mathrm{C}-$ H. .O-type intramolecular contacts. As a result of the absence of hydrogenbond donors in the structure, the crystal packing is controlled by van der Waals forces and weak $\mathrm{C}-\mathrm{H} \cdots \mathrm{O}$ interactions, which associate the molecules into inversion dimers.

\section{Chemical context}

Arylidene Meldrum's acids (5-arylmethylidene-2,2-dimethyl1,3-dioxane-4,6-diones) are attractive building blocks in organic chemistry: these compounds are used for the synthesis of different heterocycles. Recent examples include: pyrazolidinones (Pair et al., 2014), lactames (Zhang et al., 2013), carbocycles (e.g. Trost \& Maruniak, 2013) and aliphatic compounds (e.g. Mohite \& Bhat, 2013). Arylidene Meldrum's acids can be easily converted to arylmethyl Meldrum's acids [for a description of a typical procedure, see Mierina et al. (2015)], which serve as starting compounds for the synthesis of various valuable compounds [for a mini-review, see Mierina (2014)]. Apart from their wide application in syntheses, these derivatives of Meldrum's acid have been studied as platelet aggregation inhibitors (El Maatougui et al., 2012), antimalarial agents and anti-oxidants (Sandhu et al., 2010) and photostable UV-filters for cosmetic applications (Habeck \& Krause, 1999).<smiles>CCN(CC)c1ccc(C=C2C(=O)OC(C)(C)OC2=O)cc1</smiles>

\section{Structural commentary}

The title compound, $\mathrm{C}_{17} \mathrm{H}_{21} \mathrm{NO}_{4}$, consists of substituted Meldrum's acid with a [4-(diethylamino)phenyl]methylidene fragment attached to fifth position (Fig. 1.). The heterocycle assumes a distorted boat conformation. Atoms C2 and C5 


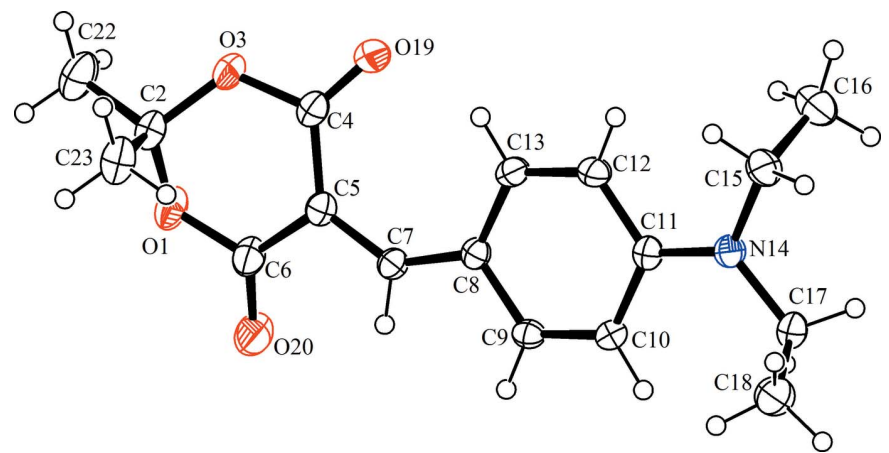

Figure 1

The molecular structure the title compound, showing $50 \%$ probability displacement ellipsoids and the atomic numbering

deviate from the least-squares plane [maximum deviations \pm 0.013 (1) $\AA$ ] calculated for the other four atoms of the heterocycle by 0.549 (3) and 0.154 (3) A, respectively. The planar part of heterocycle is nearly coplanar with the benzene ring [ dihedral angle $=8.05(10)^{\circ}$ ] due to the presence of a long conjugated system in the molecule. This leads to the formation of $\mathrm{C}-\mathrm{H}$... O-type intramolecular contacts (Table 1).

$\pi-\pi$ stacking interactions are also observed between conjugated systems of the molecules. The distance between the corresponding least-square planes is 3.54 (su?) $\AA$.

The crystal structure of the zwitterionic form of 5-[4-(diethylamino)benzyl]-2,2-dimethyl-1,3-dioxane-4,6-dione has

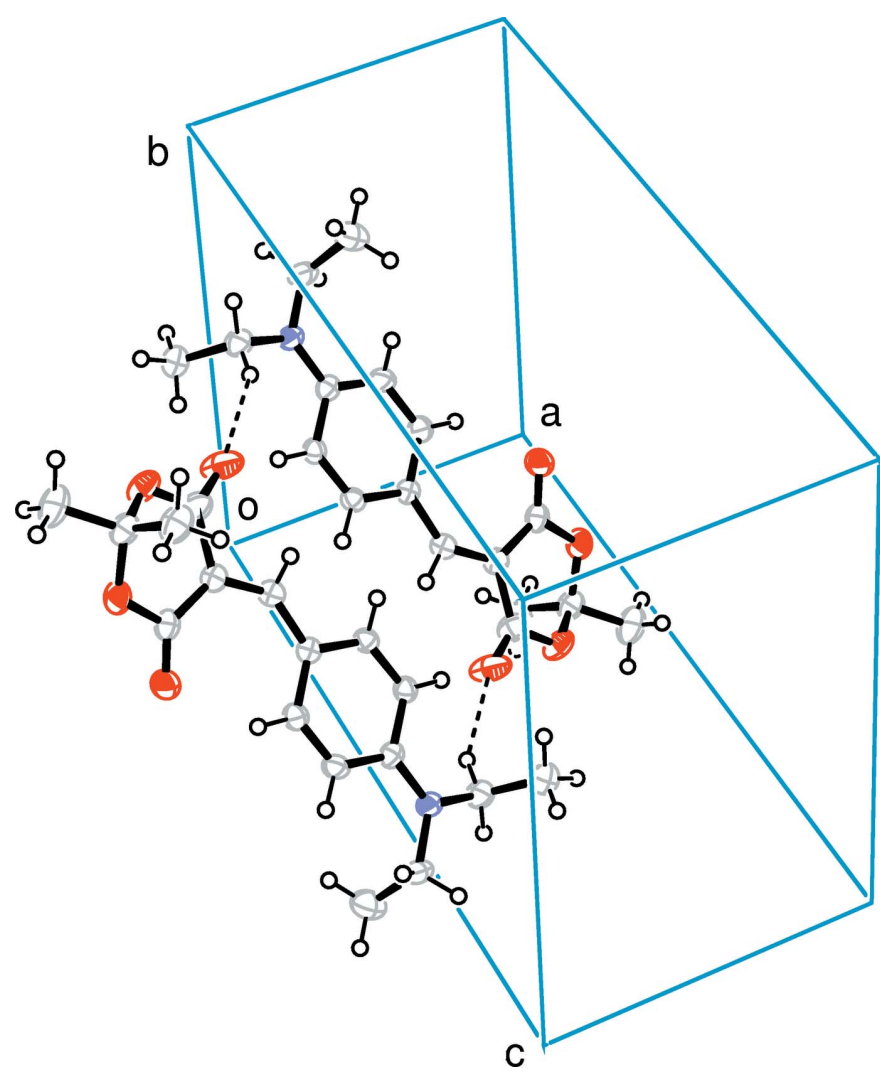

Figure 2

The crystal packing of the title compound, viewed along the $b$ axis. Hydrogen bonds are shown as dashed lines (see Table 1 for details).
Table 1

Hydrogen-bond geometry $\left(\AA,^{\circ}\right)$.

\begin{tabular}{lllll}
\hline$D-\mathrm{H} \cdots A$ & $D-\mathrm{H}$ & $\mathrm{H} \cdots A$ & $D \cdots A$ & $D-\mathrm{H} \cdots A$ \\
\hline $\mathrm{C} 13-\mathrm{H} 13 \cdots \mathrm{O} 19$ & 0.93 & 2.13 & $2.915(2)$ & 141 \\
$\mathrm{C} 17-\mathrm{H} 17 B \cdots \mathrm{O} 20^{\mathrm{i}}$ & 0.97 & 2.39 & $3.268(3)$ & 151 \\
\hline
\end{tabular}

Symmetry code: (i) $-x,-y+1,-z+1$.

been already reported (Mierina et al., 2015). The title compound differs from this by the presence of a double bond between atoms $\mathrm{C} 5$ and $\mathrm{C} 7$.

\section{Supramolecular features}

Because of the absence of hydrogen-bond donors in the structure, the crystal packing is controlled by van der Waals forces and weak $\mathrm{C}-\mathrm{H} \cdots \mathrm{O}$ interactions, which associate molecules into inversion dimers (Fig. 2, Table 1).

\section{Database survey}

Several 5-arylidene-2,2-dimethyl-1,3-dioxane-4,6-diones (Huck et al., 1995; Gould et al., 1998; Novoa de Armas et al., 2000; O'Leary et al., 2001; O'Leary \& Wallis 2006; Crawford \& McNab, 2009; Wilsily \& Fillion, 2009; Zeng, 2010a,b, 2011a,b,c, 2013; Jie, 2012; García-Álvarez et al., 2013; Dey et al., 2015) and their spiro-analogues (Sato et al., 1989; Zeng, 2011d,e,f; Zeng et al. 2013) have been characterized by X-ray analysis. However, information on the crystal structure of 5-arylmethylidene-2,2-dimethyl-1,3-dioxane-4,6-diones containing an amino functionality on the aromatic ring is not available.

\section{Synthesis and crystallization}

5-[4-(Diethylamino)phenylmethylidene]-2,2-dimethyl-1,3dioxane-4,6-dione was obtained from Meldrum's acid (1.00 g, $6.9 \mathrm{mmol})$ and 4-diethylaminobenzaldehyde $\quad(1.27 \mathrm{~g}$, $6.9 \mathrm{mmol})$ by heating in water $(50 \mathrm{ml})$ at $348 \mathrm{~K}$ for $2 \mathrm{~h}$, followed by cooling to room temperature and filtration of the formed precipitate and recrystallization from ethanol $(1.62 \mathrm{~g}$, $80 \%$ ) analogously to the method described previously (Mierina et al., 2015). The spectroscopic and physical data correspond to those in the literature (Mierina et al., 2015). $\mathrm{X}$-ray quality single crystals were obtained by slow evaporation from ethanol.

\section{Refinement}

Crystal data, data collection and structure refinement details are summarized in Table 2. The $\mathrm{C}$-bound $\mathrm{H}$ atoms were positioned geometrically and refined as riding on their parent atoms: $\mathrm{C}-\mathrm{H}=0.93-0.98 \AA$ with $U_{\text {iso }}(\mathrm{H})=1.5 U_{\text {eq }}(\mathrm{C})$ for methyl $\mathrm{H}$ atoms and $1.2 U_{\mathrm{eq}}(\mathrm{C})$ for other $\mathrm{H}$ atoms. 


\section{Acknowledgements}

IM thanks the European Social Fund for a scholarship within the project 'Support for the implementation of doctoral studies at Riga Technical University'.

\section{References}

Burla, M. C., Caliandro, R., Camalli, M., Carrozzini, B., Cascarano, G. L., Giacovazzo, C., Mallamo, M., Mazzone, A., Polidori, G. \& Spagna, R. (2012). J. Appl. Cryst. 45, 357-361.

Crawford, L. A. \& McNab, H. (2009). Collect. Czech. Chem. Commun. 74, 995-1009.

Dey, T., Ghosh, S., Ghosh, S. \& Mukherjee, A. K. (2015). J. Mol. Struct. 1092, 51-62.

El Maatougui, A., JhonnyAzuaje, B. S. P., Coelho, A., Cano, E., Yanez, M., Lopez, C., Yaziji, V., Carbajales, C. \& Sotelo, E. (2012). Combin. Chem. High Throughput Screen. 15, 551-554.

Farrugia, L. J. (2012). J. Appl. Cryst. 45, 849-854.

García-Álvarez, F., Romero, N., Lobato-García, C. E., Terán, J. L. \& Mendoza, A. (2013). Acta Cryst. E69, o50.

Gould, R. O., Harris, S. G., McNab, H., Parsons, S. \& Withell, K. (1998). Acta Cryst. C54, 234-236.

Habeck, T. \& Krause, A. (1999). German Patent 19806241.

Huck, N. P. M., Meetsma, A., Zijlstra, R. \& Feringa, B. L. (1995). Tetrahedron Lett. 36, 9381-9384.

Jie, Y. (2012). Z. Kristallogr. New Cryst. Struct. 227, 347-348.

Mierina, I. (2014). Synlett. 25, 155-156.

Mierina, I., Mishnev, A. \& Jure, M. (2015). Acta Cryst. C71, 752-758.

Mohite, A. \& Bhat, R. G. (2013). Org. Lett. 15, 4564-4567.

Nonius (1997). KappaCCD Server Software. Nonius BV, Delft, The Netherlands.

Novoa de Armas, H., Blaton, N. M., Peeters, O. M., De Ranter, C. J., Suárez, M., Ochoa, E., Verdecia, Y. \& Salfrán, E. (2000). J. Chem. Crystallogr. 30, 189-194.

O'Leary, J., Bell, P. C., Wallis, J. D. \& Schweizer, W. B. (2001). J. Chem. Soc. Perkin Trans. 2, pp. 133-139.

O'Leary, J. \& Wallis, J. D. (2006). Chem. Eur. J. 12, 7724-7732.

Otwinovski, Z. \& Minor, W. (1997). Methods in Enzymology, Vol. 276, Macromolecular Crystallography, Part A, edited by C. W. Carter Jr \& R. M. Sweet, pp. 307-326. New York: Academic Press.

Pair, E., Berini, C., Noël, R., Sanselme, M., Levacher, V. \& Brière, J.-F. (2014). Chem. Commun. 50, 10218-10221.

Sandhu, H. S., Sapra, S., Gupta, M., Nepali, K., Gautam, R., Yadav, S., Kumar, R., Jachak, S. M., Chugh, M., Gupta, M. K., Suri, O. P. \& Dhar, K. L. (2010). Bioorg. Med. Chem. 18, 5626-5633.

Sato, M., Hisamichi, H., Kaneko, C., Suzaki, N., Furuya, T. \& Inukai, N. (1989). Tetrahedron Lett. 30, 5281-5284.

Sheldrick, G. M. (2008). Acta Cryst. A64, 112-122.
Table 2

Experimental details.

\begin{tabular}{|c|c|}
\hline \multicolumn{2}{|l|}{ Crystal data } \\
\hline Chemical formula & $\mathrm{C}_{17} \mathrm{H}_{21} \mathrm{NO}_{4}$ \\
\hline$M_{\mathrm{r}}$ & 303.35 \\
\hline Crystal system, space group & Monoclinic, $P 2_{1} / c$ \\
\hline Temperature $(\mathrm{K})$ & 173 \\
\hline$a, b, c(\AA)$ & $7.8662(2), 11.4601(3), 18.1517(6)$ \\
\hline$\beta\left({ }^{\circ}\right)$ & $96.858(1)$ \\
\hline$V\left(\mathrm{~A}^{3}\right)$ & $1624.62(8)$ \\
\hline$Z$ & 4 \\
\hline Radiation type & Mo $K \alpha$ \\
\hline$\mu\left(\mathrm{mm}^{-1}\right)$ & 0.09 \\
\hline Crystal size $(\mathrm{mm})$ & $0.26 \times 0.19 \times 0.09$ \\
\hline \multicolumn{2}{|l|}{ Data collection } \\
\hline Diffractometer & Nonius KappaCCD \\
\hline $\begin{array}{l}\text { No. of measured, independent and } \\
\text { observed }[I>2 \sigma(I)] \text { reflections }\end{array}$ & $6627,3705,2183$ \\
\hline$R_{\mathrm{int}}$ & 0.054 \\
\hline$(\sin \theta / \lambda)_{\max }\left(\AA^{-1}\right)$ & 0.649 \\
\hline \multicolumn{2}{|l|}{ Refinement } \\
\hline$R\left[F^{2}>2 \sigma\left(F^{2}\right)\right], w R\left(F^{2}\right), S$ & $0.055,0.127,1.00$ \\
\hline No. of reflections & 3705 \\
\hline No. of parameters & 203 \\
\hline $\mathrm{H}$-atom treatment & $\mathrm{H}$-atom parameters constrained \\
\hline$\Delta \rho_{\max }, \Delta \rho_{\min }\left(\mathrm{e} \AA^{-3}\right)$ & $0.18,-0.19$ \\
\hline
\end{tabular}

Computer programs: KappaCCD Server Software (Nonius, 1997), HKL DENZO and SCALEPACK (Otwinovski \& Minor, 1997), SIR2011 (Burla et al., 2012), ORTEP-3 for Windows (Farrugia, 2012), SHELXL97 (Sheldrick, 2008), PLATON (Spek, 2009) and publCIF (Westrip, 2010).

Spek, A. L. (2009). Acta Cryst. D65, 148-155.

Trost, B. M. \& Maruniak, A. (2013). Angew. Chem. Int. Ed. 52, 62626264.

Westrip, S. P. (2010). J. Appl. Cryst. 43, 920-925.

Wilsily, A. \& Fillion, E. (2009). J. Org. Chem. 74, 8583-8594.

Zeng, W.-L. (2010a). Acta Cryst. E66, o2319.

Zeng, W.-L. (2010b). Acta Cryst. E66, o2366.

Zeng, W.-L. (2011a). Acta Cryst. E67, o276.

Zeng, W.-L. (2011b). Acta Cryst. E67, o1351.

Zeng, W.-L. (2011c). Acta Cryst. E67, o1937.

Zeng, W. (2011d). Asian J. Chem. 23, 4145-4147.

Zeng, W.-L. (2011e). Acta Cryst. E67, o426.

Zeng, W.-L. (2011f). Acta Cryst. E67, o1362.

Zeng, W. (2013). Asian J. Chem. 25, 864-866.

Zeng, W., Li, Y. \& Guo, H. (2013). J. Chem. Crystallogr. 43, 223-227.

Zhang, J.-P., Ding, J., Ma, N., Jiang, B., Xu, L.-C. \& Tu, S.-J. (2013). J. Hetercycl. Chem. 50, 66-70. 


\section{supporting information}

Acta Cryst. (2015). E71, 1242-1244 [doi:10.1107/S2056989015017673]

\section{Crystal structure of 5-[4-(diethylamino)benzylidene]-2,2-dimethyl-1,3- dioxane-4,6-dione}

\section{Egija Stepina, Dmitrijs Stepanovs, Inese Mierina and Mara Jure}

\section{Computing details}

Data collection: KappaCCD Server Software (Nonius, 1997); cell refinement: HKL SCALEPACK (Otwinovski \& Minor, 1997); data reduction: HKL DENZO and SCALEPACK (Otwinovski \& Minor, 1997); program(s) used to solve structure: SIR2011 (Burla et al., 2012); program(s) used to refine structure: SHELXL97 (Sheldrick, 2008); molecular graphics: ORTEP-3 for Windows (Farrugia, 2012); software used to prepare material for publication: SHELXL97 (Sheldrick, 2008), PLATON (Spek, 2009) and publCIF (Westrip, 2010).

\section{5-[4-(Diethylamino)benzylidene]-2,2-dimethyl-1,3-dioxane-4,6-dione}

Crystal data

$\mathrm{C}_{17} \mathrm{H}_{21} \mathrm{NO}_{4}$

$M_{r}=303.35$

Monoclinic, $P 2_{1} / c$

Hall symbol: $-\mathrm{P} 2 \mathrm{ybc}$

$a=7.8662(2) \AA$

$b=11.4601(3) \AA$

$c=18.1517(6) \AA$

$\beta=96.858(1)^{\circ}$

$V=1624.62(8) \AA^{3}$

$Z=4$

\section{Data collection}

Nonius KappaCCD diffractometer

Radiation source: fine-focus sealed tube Graphite monochromator

CCD scans

6627 measured reflections

3705 independent reflections

Refinement

Refinement on $F^{2}$

Least-squares matrix: full

$R\left[F^{2}>2 \sigma\left(F^{2}\right)\right]=0.055$

$w R\left(F^{2}\right)=0.127$

$S=1.00$

3705 reflections

203 parameters

0 restraints
$F(000)=648$

$D_{\mathrm{x}}=1.240 \mathrm{Mg} \mathrm{m}^{-3}$

Mo $K \alpha$ radiation, $\lambda=0.71073 \AA$

Cell parameters from 15405 reflections

$\theta=1.0-27.5^{\circ}$

$\mu=0.09 \mathrm{~mm}^{-1}$

$T=173 \mathrm{~K}$

Plate, red

$0.26 \times 0.19 \times 0.09 \mathrm{~mm}$

2183 reflections with $I>2 \sigma(I)$

$R_{\text {int }}=0.054$

$\theta_{\max }=27.5^{\circ}, \theta_{\min }=2.3^{\circ}$

$h=-10 \rightarrow 10$

$k=-14 \rightarrow 13$

$l=-23 \rightarrow 23$

Primary atom site location: structure-invariant direct methods

Secondary atom site location: difference Fourier map

Hydrogen site location: inferred from neighbouring sites

$\mathrm{H}$-atom parameters constrained 
$w=1 /\left[\sigma^{2}\left(F_{\mathrm{o}}^{2}\right)+(0.0534 P)^{2}+0.0774 P\right]$

where $P=\left(F_{\mathrm{o}}^{2}+2 F_{\mathrm{c}}^{2}\right) / 3$

$(\Delta / \sigma)_{\max }<0.001$

$$
\Delta \rho_{\max }=0.18 \mathrm{e} \AA^{-3}
$$$$
\Delta \rho_{\min }=-0.19 \mathrm{e} \AA^{-3}
$$

\section{Special details}

Geometry. All e.s.d.'s (except the e.s.d. in the dihedral angle between two l.s. planes) are estimated using the full covariance matrix. The cell e.s.d.'s are taken into account individually in the estimation of e.s.d.'s in distances, angles and torsion angles; correlations between e.s.d.'s in cell parameters are only used when they are defined by crystal symmetry. An approximate (isotropic) treatment of cell e.s.d.'s is used for estimating e.s.d.'s involving 1.s. planes.

Refinement. Refinement of $F^{2}$ against ALL reflections. The weighted $R$-factor $w R$ and goodness of fit $S$ are based on $F^{2}$, conventional $R$-factors $R$ are based on $F$, with $F$ set to zero for negative $F^{2}$. The threshold expression of $F^{2}>\sigma\left(F^{2}\right)$ is used only for calculating $R$-factors(gt) etc. and is not relevant to the choice of reflections for refinement. $R$-factors based on $F^{2}$ are statistically about twice as large as those based on $F$, and $R$ - factors based on ALL data will be even larger.

Fractional atomic coordinates and isotropic or equivalent isotropic displacement parameters $\left(\AA^{2}\right)$

\begin{tabular}{|c|c|c|c|c|}
\hline & $x$ & $y$ & $z$ & $U_{\text {iso }} * / U_{\text {eq }}$ \\
\hline $\mathrm{O} 1$ & $0.57779(16)$ & $0.16291(12)$ & $0.51388(7)$ & $0.0404(4)$ \\
\hline O19 & $0.65514(15)$ & $0.45154(13)$ & $0.40080(8)$ & $0.0407(4)$ \\
\hline $\mathrm{O} 3$ & $0.73309(14)$ & $0.27588(11)$ & $0.43789(8)$ & $0.0390(4)$ \\
\hline $\mathrm{C} 8$ & $0.2432(2)$ & $0.50423(15)$ & $0.40398(10)$ & $0.0252(4)$ \\
\hline $\mathrm{C} 10$ & $-0.0015(2)$ & $0.63516(16)$ & $0.37433(10)$ & $0.0266(4)$ \\
\hline H10 & -0.1138 & 0.6545 & 0.3804 & $0.032 *$ \\
\hline $\mathrm{O} 20$ & $0.33160(19)$ & $0.21986(13)$ & $0.54631(9)$ & $0.0584(5)$ \\
\hline N14 & $0.02179(17)$ & $0.80169(13)$ & $0.29384(9)$ & $0.0307(4)$ \\
\hline $\mathrm{C} 12$ & $0.2607(2)$ & $0.66959(17)$ & $0.32158(11)$ & $0.0317(5)$ \\
\hline H12 & 0.3242 & 0.7124 & 0.2910 & $0.038^{*}$ \\
\hline $\mathrm{C} 9$ & $0.0729(2)$ & $0.53919(16)$ & $0.40930(10)$ & $0.0259(4)$ \\
\hline $\mathrm{H} 9$ & 0.0078 & 0.4944 & 0.4382 & $0.031^{*}$ \\
\hline C11 & $0.0910(2)$ & $0.70529(16)$ & $0.32900(10)$ & $0.0266(4)$ \\
\hline $\mathrm{C} 4$ & $0.6131(2)$ & $0.36263(18)$ & $0.42864(11)$ & $0.0310(5)$ \\
\hline C13 & $0.3340(2)$ & $0.57427(17)$ & $0.35789(11)$ & $0.0309(5)$ \\
\hline H13 & 0.4465 & 0.5550 & 0.3521 & $0.037^{*}$ \\
\hline $\mathrm{C} 7$ & $0.3028(2)$ & $0.40397(16)$ & $0.44569(10)$ & $0.0275(4)$ \\
\hline $\mathrm{H} 7$ & 0.2188 & 0.3745 & 0.4725 & $0.033^{*}$ \\
\hline $\mathrm{C} 15$ & $0.1135(2)$ & $0.86883(18)$ & $0.24297(11)$ & $0.0386(5)$ \\
\hline $\mathrm{H} 15 \mathrm{~A}$ & 0.0311 & 0.9075 & 0.2070 & $0.046^{*}$ \\
\hline H15B & 0.1801 & 0.8159 & 0.2161 & $0.046^{*}$ \\
\hline $\mathrm{C} 5$ & $0.4505(2)$ & $0.33863(16)$ & $0.45730(10)$ & $0.0292(4)$ \\
\hline C6 & $0.4436(3)$ & $0.23885(18)$ & $0.50801(11)$ & $0.0377(5)$ \\
\hline $\mathrm{C} 2$ & $0.6814(2)$ & $0.16029(17)$ & $0.45458(12)$ & $0.0363(5)$ \\
\hline C18 & $-0.2946(2)$ & $0.7988(2)$ & $0.25955(13)$ & $0.0473(6)$ \\
\hline $\mathrm{H} 18 \mathrm{~A}$ & -0.2752 & 0.8057 & 0.2085 & $0.071 *$ \\
\hline H18B & -0.3967 & 0.8406 & 0.2673 & $0.071^{*}$ \\
\hline $\mathrm{H} 18 \mathrm{C}$ & -0.3077 & 0.7180 & 0.2716 & $0.071^{*}$ \\
\hline $\mathrm{C} 17$ & $-0.1429(2)$ & $0.84969(17)$ & $0.30887(12)$ & $0.0349(5)$ \\
\hline H17A & -0.1415 & 0.9336 & 0.3019 & $0.042 *$ \\
\hline H17B & -0.1577 & 0.8348 & 0.3603 & $0.042 *$ \\
\hline $\mathrm{C} 21$ & $0.8427(3)$ & $0.0962(2)$ & $0.48418(14)$ & $0.0551(6)$ \\
\hline
\end{tabular}


supporting information

\begin{tabular}{lllll}
$\mathrm{H} 21 \mathrm{~A}$ & 0.8967 & 0.1357 & 0.5274 & $0.083^{*}$ \\
$\mathrm{H} 21 \mathrm{~B}$ & 0.9195 & 0.0943 & 0.4469 & $0.083^{*}$ \\
$\mathrm{H} 21 \mathrm{C}$ & 0.8146 & 0.0179 & 0.4971 & $0.083^{*}$ \\
$\mathrm{C} 22$ & $0.5872(3)$ & $0.1025(2)$ & $0.38720(12)$ & $0.0460(6)$ \\
$\mathrm{H} 22 \mathrm{~A}$ & 0.5555 & 0.0247 & 0.3997 & $0.069^{*}$ \\
$\mathrm{H} 22 \mathrm{~B}$ & 0.6600 & 0.0994 & 0.3484 & $0.069^{*}$ \\
$\mathrm{H} 22 \mathrm{C}$ & 0.4860 & 0.1465 & 0.3706 & $0.069^{*}$ \\
$\mathrm{C} 16$ & $0.2319(3)$ & $0.9596(2)$ & $0.28194(14)$ & $0.0533(6)$ \\
$\mathrm{H} 16 \mathrm{~A}$ & 0.1656 & 1.0166 & 0.3047 & $0.080^{*}$ \\
$\mathrm{H} 16 \mathrm{~B}$ & 0.2950 & 0.9971 & 0.2465 & $0.080^{*}$ \\
$\mathrm{H} 16 \mathrm{C}$ & 0.3102 & 0.9224 & 0.3194 & $0.080^{*}$ \\
\hline
\end{tabular}

Atomic displacement parameters $\left(\AA^{2}\right)$

\begin{tabular}{lllllll}
\hline & $U^{11}$ & $U^{22}$ & $U^{33}$ & $U^{12}$ & $U^{13}$ & $U^{23}$ \\
\hline O1 & $0.0464(8)$ & $0.0385(9)$ & $0.0367(8)$ & $0.0179(6)$ & $0.0058(6)$ & $0.0069(7)$ \\
O19 & $0.0303(7)$ & $0.0382(9)$ & $0.0546(10)$ & $0.0026(6)$ & $0.0093(6)$ & $0.0078(7)$ \\
O3 & $0.0274(7)$ & $0.0354(8)$ & $0.0538(9)$ & $0.0093(6)$ & $0.0033(6)$ & $0.0008(7)$ \\
C8 & $0.0267(9)$ & $0.0252(10)$ & $0.0236(10)$ & $0.0011(7)$ & $0.0025(7)$ & $-0.0030(8)$ \\
C10 & $0.0229(9)$ & $0.0296(11)$ & $0.0276(10)$ & $0.0014(7)$ & $0.0039(7)$ & $-0.0005(9)$ \\
O20 & $0.0643(10)$ & $0.0510(11)$ & $0.0666(11)$ & $0.0218(8)$ & $0.0352(9)$ & $0.0286(9)$ \\
N14 & $0.0289(8)$ & $0.0294(9)$ & $0.0341(10)$ & $0.0030(7)$ & $0.0042(7)$ & $0.0077(7)$ \\
C12 & $0.0287(9)$ & $0.0320(12)$ & $0.0357(12)$ & $-0.0004(8)$ & $0.0096(8)$ & $0.0068(9)$ \\
C9 & $0.0263(9)$ & $0.0292(11)$ & $0.0229(10)$ & $-0.0026(7)$ & $0.0058(7)$ & $-0.0004(8)$ \\
C11 & $0.0291(9)$ & $0.0257(10)$ & $0.0241(10)$ & $0.0014(8)$ & $-0.0003(7)$ & $-0.0027(8)$ \\
C4 & $0.0285(10)$ & $0.0321(12)$ & $0.0315(11)$ & $0.0051(8)$ & $0.0000(8)$ & $-0.0043(10)$ \\
C13 & $0.0243(9)$ & $0.0335(11)$ & $0.0359(12)$ & $0.0028(8)$ & $0.0077(8)$ & $0.0029(9)$ \\
C7 & $0.0292(9)$ & $0.0266(11)$ & $0.0279(11)$ & $0.0007(8)$ & $0.0082(7)$ & $-0.0034(9)$ \\
C15 & $0.0417(11)$ & $0.0358(12)$ & $0.0389(12)$ & $0.0026(9)$ & $0.0070(9)$ & $0.0156(10)$ \\
C5 & $0.0303(9)$ & $0.0279(11)$ & $0.0293(11)$ & $0.0026(8)$ & $0.0033(7)$ & $-0.0022(9)$ \\
C6 & $0.0430(11)$ & $0.0346(12)$ & $0.0364(12)$ & $0.0103(9)$ & $0.0086(9)$ & $0.0034(10)$ \\
C2 & $0.0360(11)$ & $0.0324(12)$ & $0.0403(13)$ & $0.0108(9)$ & $0.0033(9)$ & $0.0005(10)$ \\
C18 & $0.0347(11)$ & $0.0472(14)$ & $0.0580(15)$ & $0.0033(9)$ & $-0.0029(10)$ & $0.0115(12)$ \\
C17 & $0.0342(10)$ & $0.0286(11)$ & $0.0421(12)$ & $0.0069(8)$ & $0.0057(8)$ & $0.0037(10)$ \\
C21 & $0.0446(12)$ & $0.0548(16)$ & $0.0634(17)$ & $0.0220(11)$ & $-0.0038(11)$ & $0.0016(13)$ \\
C22 & $0.0522(12)$ & $0.0397(14)$ & $0.0441(14)$ & $0.0123(10)$ & $-0.0023(10)$ & $-0.0057(11)$ \\
C16 & $0.0518(13)$ & $0.0419(14)$ & $0.0670(17)$ & $-0.0090(10)$ & $0.0103(11)$ & $0.0091(12)$ \\
& & & & & & \\
\hline & & & & & & \\
\hline
\end{tabular}

Geometric parameters $\left(A,{ }^{\circ}\right)$

\begin{tabular}{llll}
\hline $\mathrm{O} 1-\mathrm{C} 6$ & $1.363(2)$ & $\mathrm{C} 7-\mathrm{H} 7$ & 0.9300 \\
$\mathrm{O} 1-\mathrm{C} 2$ & $1.426(2)$ & $\mathrm{C} 15-\mathrm{C} 16$ & $1.514(3)$ \\
$\mathrm{O} 19-\mathrm{C} 4$ & $1.201(2)$ & $\mathrm{C} 15-\mathrm{H} 15 \mathrm{~A}$ & 0.9700 \\
$\mathrm{O} 3-\mathrm{C} 4$ & $1.368(2)$ & $\mathrm{C} 15-\mathrm{H} 15 \mathrm{~B}$ & 0.9700 \\
$\mathrm{O} 3-\mathrm{C} 2$ & $1.429(2)$ & $\mathrm{C} 5-\mathrm{C} 6$ & $1.473(3)$ \\
$\mathrm{C} 8-\mathrm{C} 9$ & $1.413(2)$ & $\mathrm{C} 2-\mathrm{C} 22$ & $1.506(3)$ \\
$\mathrm{C} 8-\mathrm{C} 13$ & $1.413(2)$ & $\mathrm{C} 2-\mathrm{C} 21$ & $1.509(3)$ \\
$\mathrm{C} 8-\mathrm{C} 7$ & $1.424(2)$ & $\mathrm{C} 18-\mathrm{C} 17$ & $1.519(3)$
\end{tabular}




\begin{tabular}{|c|c|c|c|}
\hline $\mathrm{C} 10-\mathrm{C} 9$ & $1.366(2)$ & $\mathrm{C} 18-\mathrm{H} 18 \mathrm{~A}$ & 0.9600 \\
\hline $\mathrm{C} 10-\mathrm{C} 11$ & $1.413(3)$ & C18-H18B & 0.9600 \\
\hline $\mathrm{C} 10-\mathrm{H} 10$ & 0.9300 & $\mathrm{C} 18-\mathrm{H} 18 \mathrm{C}$ & 0.9600 \\
\hline $\mathrm{O} 20-\mathrm{C} 6$ & $1.205(2)$ & C17-H17A & 0.9700 \\
\hline $\mathrm{N} 14-\mathrm{C} 11$ & $1.357(2)$ & C17-H17B & 0.9700 \\
\hline $\mathrm{N} 14-\mathrm{C} 15$ & $1.457(2)$ & $\mathrm{C} 21-\mathrm{H} 21 \mathrm{~A}$ & 0.9600 \\
\hline $\mathrm{N} 14-\mathrm{C} 17$ & $1.463(2)$ & $\mathrm{C} 21-\mathrm{H} 21 \mathrm{~B}$ & 0.9600 \\
\hline $\mathrm{C} 12-\mathrm{C} 13$ & $1.367(3)$ & $\mathrm{C} 21-\mathrm{H} 21 \mathrm{C}$ & 0.9600 \\
\hline $\mathrm{C} 12-\mathrm{C} 11$ & $1.418(2)$ & $\mathrm{C} 22-\mathrm{H} 22 \mathrm{~A}$ & 0.9600 \\
\hline $\mathrm{C} 12-\mathrm{H} 12$ & 0.9300 & $\mathrm{C} 22-\mathrm{H} 22 \mathrm{~B}$ & 0.9600 \\
\hline $\mathrm{C} 9-\mathrm{H} 9$ & 0.9300 & $\mathrm{C} 22-\mathrm{H} 22 \mathrm{C}$ & 0.9600 \\
\hline $\mathrm{C} 4-\mathrm{C} 5$ & $1.463(2)$ & $\mathrm{C} 16-\mathrm{H} 16 \mathrm{~A}$ & 0.9600 \\
\hline $\mathrm{C} 13-\mathrm{H} 13$ & 0.9300 & C16-H16B & 0.9600 \\
\hline $\mathrm{C} 7-\mathrm{C} 5$ & $1.377(2)$ & $\mathrm{C} 16-\mathrm{H} 16 \mathrm{C}$ & 0.9600 \\
\hline $\mathrm{C} 6-\mathrm{O} 1-\mathrm{C} 2$ & $117.50(15)$ & $\mathrm{O} 20-\mathrm{C} 6-\mathrm{C} 5$ & $125.78(18)$ \\
\hline $\mathrm{C} 4-\mathrm{O} 3-\mathrm{C} 2$ & $119.35(14)$ & $\mathrm{O} 1-\mathrm{C} 6-\mathrm{C} 5$ & $117.29(17)$ \\
\hline $\mathrm{C} 9-\mathrm{C} 8-\mathrm{C} 13$ & $115.46(16)$ & $\mathrm{O} 1-\mathrm{C} 2-\mathrm{O} 3$ & $110.09(15)$ \\
\hline $\mathrm{C} 9-\mathrm{C} 8-\mathrm{C} 7$ & $116.56(16)$ & $\mathrm{O} 1-\mathrm{C} 2-\mathrm{C} 22$ & $110.63(16)$ \\
\hline $\mathrm{C} 13-\mathrm{C} 8-\mathrm{C} 7$ & $127.97(16)$ & $\mathrm{O} 3-\mathrm{C} 2-\mathrm{C} 22$ & $111.13(17)$ \\
\hline $\mathrm{C} 9-\mathrm{C} 10-\mathrm{C} 11$ & $120.46(16)$ & $\mathrm{O} 1-\mathrm{C} 2-\mathrm{C} 21$ & $105.88(17)$ \\
\hline $\mathrm{C} 9-\mathrm{C} 10-\mathrm{H} 10$ & 119.8 & $\mathrm{O} 3-\mathrm{C} 2-\mathrm{C} 21$ & $106.15(16)$ \\
\hline $\mathrm{C} 11-\mathrm{C} 10-\mathrm{H} 10$ & 119.8 & $\mathrm{C} 22-\mathrm{C} 2-\mathrm{C} 21$ & $112.74(18)$ \\
\hline $\mathrm{C} 11-\mathrm{N} 14-\mathrm{C} 15$ & $121.78(15)$ & $\mathrm{C} 17-\mathrm{C} 18-\mathrm{H} 18 \mathrm{~A}$ & 109.5 \\
\hline $\mathrm{C} 11-\mathrm{N} 14-\mathrm{C} 17$ & $122.20(15)$ & $\mathrm{C} 17-\mathrm{C} 18-\mathrm{H} 18 \mathrm{~B}$ & 109.5 \\
\hline $\mathrm{C} 15-\mathrm{N} 14-\mathrm{C} 17$ & $115.86(15)$ & $\mathrm{H} 18 \mathrm{~A}-\mathrm{C} 18-\mathrm{H} 18 \mathrm{~B}$ & 109.5 \\
\hline $\mathrm{C} 13-\mathrm{C} 12-\mathrm{C} 11$ & $122.18(17)$ & $\mathrm{C} 17-\mathrm{C} 18-\mathrm{H} 18 \mathrm{C}$ & 109.5 \\
\hline $\mathrm{C} 13-\mathrm{C} 12-\mathrm{H} 12$ & 118.9 & $\mathrm{H} 18 \mathrm{~A}-\mathrm{C} 18-\mathrm{H} 18 \mathrm{C}$ & 109.5 \\
\hline $\mathrm{C} 11-\mathrm{C} 12-\mathrm{H} 12$ & 118.9 & $\mathrm{H} 18 \mathrm{~B}-\mathrm{C} 18-\mathrm{H} 18 \mathrm{C}$ & 109.5 \\
\hline $\mathrm{C} 10-\mathrm{C} 9-\mathrm{C} 8$ & $123.61(16)$ & $\mathrm{N} 14-\mathrm{C} 17-\mathrm{C} 18$ & $113.34(17)$ \\
\hline $\mathrm{C} 10-\mathrm{C} 9-\mathrm{H} 9$ & 118.2 & N14-C17-H17A & 108.9 \\
\hline $\mathrm{C} 8-\mathrm{C} 9-\mathrm{H} 9$ & 118.2 & $\mathrm{C} 18-\mathrm{C} 17-\mathrm{H} 17 \mathrm{~A}$ & 108.9 \\
\hline $\mathrm{N} 14-\mathrm{C} 11-\mathrm{C} 10$ & $122.12(16)$ & N14-C17-H17B & 108.9 \\
\hline $\mathrm{N} 14-\mathrm{C} 11-\mathrm{C} 12$ & $121.31(16)$ & $\mathrm{C} 18-\mathrm{C} 17-\mathrm{H} 17 \mathrm{~B}$ & 108.9 \\
\hline $\mathrm{C} 10-\mathrm{C} 11-\mathrm{C} 12$ & $116.57(16)$ & $\mathrm{H} 17 \mathrm{~A}-\mathrm{C} 17-\mathrm{H} 17 \mathrm{~B}$ & 107.7 \\
\hline $\mathrm{O} 19-\mathrm{C} 4-\mathrm{O} 3$ & $116.57(16)$ & $\mathrm{C} 2-\mathrm{C} 21-\mathrm{H} 21 \mathrm{~A}$ & 109.5 \\
\hline $\mathrm{O} 19-\mathrm{C} 4-\mathrm{C} 5$ & $127.27(17)$ & $\mathrm{C} 2-\mathrm{C} 21-\mathrm{H} 21 \mathrm{~B}$ & 109.5 \\
\hline $\mathrm{O} 3-\mathrm{C} 4-\mathrm{C} 5$ & $116.09(17)$ & $\mathrm{H} 21 \mathrm{~A}-\mathrm{C} 21-\mathrm{H} 21 \mathrm{~B}$ & 109.5 \\
\hline $\mathrm{C} 12-\mathrm{C} 13-\mathrm{C} 8$ & $121.68(16)$ & $\mathrm{C} 2-\mathrm{C} 21-\mathrm{H} 21 \mathrm{C}$ & 109.5 \\
\hline $\mathrm{C} 12-\mathrm{C} 13-\mathrm{H} 13$ & 119.2 & $\mathrm{H} 21 \mathrm{~A}-\mathrm{C} 21-\mathrm{H} 21 \mathrm{C}$ & 109.5 \\
\hline $\mathrm{C} 8-\mathrm{C} 13-\mathrm{H} 13$ & 119.2 & $\mathrm{H} 21 \mathrm{~B}-\mathrm{C} 21-\mathrm{H} 21 \mathrm{C}$ & 109.5 \\
\hline $\mathrm{C} 5-\mathrm{C} 7-\mathrm{C} 8$ & $137.58(17)$ & $\mathrm{C} 2-\mathrm{C} 22-\mathrm{H} 22 \mathrm{~A}$ & 109.5 \\
\hline $\mathrm{C} 5-\mathrm{C} 7-\mathrm{H} 7$ & 111.2 & $\mathrm{C} 2-\mathrm{C} 22-\mathrm{H} 22 \mathrm{~B}$ & 109.5 \\
\hline $\mathrm{C} 8-\mathrm{C} 7-\mathrm{H} 7$ & 111.2 & $\mathrm{H} 22 \mathrm{~A}-\mathrm{C} 22-\mathrm{H} 22 \mathrm{~B}$ & 109.5 \\
\hline $\mathrm{N} 14-\mathrm{C} 15-\mathrm{C} 16$ & $112.95(18)$ & $\mathrm{C} 2-\mathrm{C} 22-\mathrm{H} 22 \mathrm{C}$ & 109.5 \\
\hline $\mathrm{N} 14-\mathrm{C} 15-\mathrm{H} 15 \mathrm{~A}$ & 109.0 & $\mathrm{H} 22 \mathrm{~A}-\mathrm{C} 22-\mathrm{H} 22 \mathrm{C}$ & 109.5 \\
\hline $\mathrm{C} 16-\mathrm{C} 15-\mathrm{H} 15 \mathrm{~A}$ & 109.0 & $\mathrm{H} 22 \mathrm{~B}-\mathrm{C} 22-\mathrm{H} 22 \mathrm{C}$ & 109.5 \\
\hline N14-C15-H15B & 109.0 & $\mathrm{C} 15-\mathrm{C} 16-\mathrm{H} 16 \mathrm{~A}$ & 109.5 \\
\hline
\end{tabular}




$\begin{array}{llll}\mathrm{C} 16-\mathrm{C} 15-\mathrm{H} 15 \mathrm{~B} & 109.0 & \mathrm{C} 15-\mathrm{C} 16-\mathrm{H} 16 \mathrm{~B} & 109.5 \\ \mathrm{H} 15 \mathrm{~A}-\mathrm{C} 15-\mathrm{H} 15 \mathrm{~B} & 107.8 & \mathrm{H} 16 \mathrm{~A}-\mathrm{C} 16-\mathrm{H} 16 \mathrm{~B} & 109.5 \\ \mathrm{C} 7-\mathrm{C} 5-\mathrm{C} 4 & 126.92(18) & \mathrm{C} 15-\mathrm{C} 16-\mathrm{H} 16 \mathrm{C} & 109.5 \\ \mathrm{C} 7-\mathrm{C} 5-\mathrm{C} 6 & 115.10(16) & \mathrm{H} 16 \mathrm{~A}-\mathrm{C} 16-\mathrm{H} 16 \mathrm{C} & 109.5 \\ \mathrm{C} 4-\mathrm{C} 5-\mathrm{C} 6 & \mathrm{H} 16 \mathrm{~B}-\mathrm{C} 16-\mathrm{H} 16 \mathrm{C} & 109.5 \\ \text { O20-C6-O1 } & 117.86(16) & & \\ & 116.90(18) & \mathrm{C} 8-\mathrm{C} 7-\mathrm{C} 5-\mathrm{C} 4 & 4.4(4) \\ \mathrm{C} 11-\mathrm{C} 10-\mathrm{C} 9-\mathrm{C} 8 & 0.8(3) & \mathrm{C} 8-\mathrm{C} 7-\mathrm{C} 5-\mathrm{C} 6 & -179.8(2) \\ \mathrm{C} 13-\mathrm{C} 8-\mathrm{C} 9-\mathrm{C} 10 & -1.4(3) & \mathrm{O} 19-\mathrm{C} 4-\mathrm{C} 5-\mathrm{C} 7 & 13.2(3) \\ \mathrm{C} 7-\mathrm{C} 8-\mathrm{C} 9-\mathrm{C} 10 & 178.19(17) & \mathrm{O} 3-\mathrm{C} 4-\mathrm{C} 5-\mathrm{C} 7 & -170.14(18) \\ \mathrm{C} 15-\mathrm{N} 14-\mathrm{C} 11-\mathrm{C} 10 & -175.52(17) & \mathrm{O} 19-\mathrm{C} 4-\mathrm{C} 5-\mathrm{C} 6 & -162.49(19) \\ \mathrm{C} 17-\mathrm{N} 14-\mathrm{C} 11-\mathrm{C} 10 & 9.3(3) & \mathrm{C} 2-\mathrm{O} 1-\mathrm{C} 6-\mathrm{O} 20 & 14.2(2) \\ \mathrm{C} 15-\mathrm{N} 14-\mathrm{C} 11-\mathrm{C} 12 & 4.1(3) & \mathrm{C} 2-\mathrm{O} 1-\mathrm{C} 6-\mathrm{C} 5 & 160.50(19) \\ \mathrm{C} 17-\mathrm{N} 14-\mathrm{C} 11-\mathrm{C} 12 & -171.03(17) & \mathrm{C} 7-\mathrm{C} 5-\mathrm{C} 6-\mathrm{O} 20 & -21.3(2) \\ \mathrm{C} 9-\mathrm{C} 10-\mathrm{C} 11-\mathrm{N} 14 & -179.51(17) & \mathrm{C} 4-\mathrm{C} 5-\mathrm{C} 6-\mathrm{O} 20 & 166.3(3) \\ \mathrm{C} 9-\mathrm{C} 10-\mathrm{C} 11-\mathrm{C} 12 & 0.8(3) & \mathrm{C} 7-\mathrm{C} 5-\mathrm{C} 6-\mathrm{O} 1 & 171.75(17) \\ \mathrm{C} 13-\mathrm{C} 12-\mathrm{C} 11-\mathrm{N} 14 & 178.48(18) & \mathrm{C} 4-\mathrm{C} 5-\mathrm{C} 6-\mathrm{O} 1 & -12.0(3) \\ \mathrm{C} 13-\mathrm{C} 12-\mathrm{C} 11-\mathrm{C} 10 & -1.8(3) & \mathrm{C} 6-\mathrm{O} 1-\mathrm{C} 2-\mathrm{O} 3 & 50.5(2) \\ \mathrm{C} 2-\mathrm{O} 3-\mathrm{C} 4-\mathrm{O} 19 & -165.89(17) & \mathrm{C} 6-\mathrm{O} 1-\mathrm{C} 2-\mathrm{C} 22 & -72.7(2) \\ \mathrm{C} 2-\mathrm{O} 3-\mathrm{C} 4-\mathrm{C} 5 & 17.1(2) & \mathrm{C} 6-\mathrm{O} 1-\mathrm{C} 2-\mathrm{C} 21 & 164.84(18) \\ \mathrm{C} 11-\mathrm{C} 12-\mathrm{C} 13-\mathrm{C} 8 & 1.3(3) & \mathrm{C} 4-\mathrm{O} 3-\mathrm{C} 2-\mathrm{O} 1 & -48.8(2) \\ \mathrm{C} 9-\mathrm{C} 8-\mathrm{C} 13-\mathrm{C} 12 & 0.4(3) & \mathrm{C} 4-\mathrm{O} 3-\mathrm{C} 2-\mathrm{C} 22 & 74.2(2) \\ \mathrm{C} 7-\mathrm{C} 8-\mathrm{C} 13-\mathrm{C} 12 & -179.18(18) & \mathrm{C} 4-\mathrm{O} 3-\mathrm{C} 2-\mathrm{C} 21 & -162.94(17) \\ \mathrm{C} 9-\mathrm{C} 8-\mathrm{C} 7-\mathrm{C} 5 & 179.1(2) & \mathrm{C} 15-\mathrm{N} 14-\mathrm{C} 17-\mathrm{C} 18 & -89.7(2) \\ \mathrm{C} 13-\mathrm{C} 8-\mathrm{C} 7-\mathrm{C} 5 & -1.4(4) & -85.9(2) & 84.8(2) \\ \mathrm{C} 11-\mathrm{N} 14-\mathrm{C} 15-\mathrm{C} 16 & 89.6(2) & & \\ \mathrm{C} 17-\mathrm{N} 14-\mathrm{C} 15-\mathrm{C} 16 & & & \end{array}$

Hydrogen-bond geometry $\left(\AA,{ }^{\circ}\right)$

\begin{tabular}{lllll}
\hline$D-\mathrm{H} \cdots A$ & $D-\mathrm{H}$ & $\mathrm{H} \cdots A$ & $D \cdots A$ & $D-\mathrm{H} \cdots A$ \\
\hline $\mathrm{C} 13-\mathrm{H} 13 \cdots \mathrm{O} 19$ & 0.93 & 2.13 & $2.915(2)$ & 141 \\
$\mathrm{C} 17-\mathrm{H} 17 B \cdots \mathrm{O} 20^{\mathrm{i}}$ & 0.97 & 2.39 & $3.268(3)$ & 151 \\
\hline
\end{tabular}

Symmetry code: (i) $-x,-y+1,-z+1$. 